\title{
Oral Health Status in Children with Autism Spectrum Disorder Presenting in Yaoundé, Cameroon
}

\author{
Ashu Michael Agbor ${ }^{1 *}$, Carine Flore Moyopo Sugim ${ }^{1}$, Leonard Tedong${ }^{1}$ and Sudeshni Naidoo ${ }^{2}$ \\ ${ }^{1}$ University des Montagne's, School of Dentistry, Cameroon, Africa \\ ${ }^{2}$ Department of Community Oral health, University of the Western Cape, Cape town, South Africa \\ *Corresponding author: Ashu Michael Agbor, University des Montagne's, School of Dentistry, Cameroon, Africa
}

\begin{abstract}
Introduction: Children with Autism Spectrum Disorders (ASD) present with a lifelong neuro-developmental disorder that makes them prone to oral diseases. The aim of the present study was to describe the oral health status of children with ASD attending autism centers of Yaoundé, Cameroon.
\end{abstract}

Methodology: A descriptive cross-sectional study was conducted in seven specialized centers caring for children with autism. A structured questionnaire and clinical examinations were used to collect the data.

Results: Ninety six children aged between 3 to 14 years, the majority of whom were boys (80\%), were recruited into the study. The majority were from kindergarten (75\%) and the remainder primary schools. Tooth brushing for most of the children (71\%) was carried out by their mother. Half of the children brushed once a day with the help of their mothers usually in the morning before breakfast, using toothbrushes and toothpaste. Most of the children consumed soft and sweet diets that included sweetened fruit juices and had never been to a dentist. The prevalence of caries was $66.13 \%$. Dental caries was more pronounced in the 7 to 11 year old age group. Almost half (45.21\%) had gingivitis associated with plaque and calculus and 59.19\% had gingival bleeding. The prevalence of trauma to both hard and soft oral tissues was $45.83 \%$ while the prevalence of dental abnormalities was $31 \%$. The treatment needs of the children include dental fillings, scaling and curettage.

Conclusion: Children with ASD presented with a high prevalence of dental caries and periodontal diseases, bad breath and dental trauma to hard and soft tissues.

Recommendation: Since autism patients present with many functional and clinical problems, a multidisciplinary approach is needed in Cameroon for the management of medical, dental and behavioral problems.

Keywords: Autism Spectrum Disease; Cameroon; Children; Oral health

\section{Introduction}

Oral diseases such a dental caries and periodontal disease are the most common chronic diseases and are important public health problems because of their high prevalence [1]. Oral health and general health are interlinked, affecting and affected by one another. General health can affect oral health. Medical conditions often have oral implications and consequences [1,2] and one disease affecting general health that can affect oral health is Autism which is considered a disease of special care within the oral health care services. Results of the 1994-1995 National Health Interview
Survey of the USA on access to care and use of services indicated that the most prevalent unmet health need among children with special health care needs was oral health care [3]. During the 20142016 survey, the prevalence of children aged 3-17 years who had ever been diagnosed with a developmental disability increased from $5.76 \%$ to $6.99 \%$. During this same time, the prevalence of diagnosed autism spectrum disorder and intellectual disability did not change significantly. The prevalence of autism spectrum disorder, intellectual disability, other developmental delay, and any 
developmental disability was higher among boys when compared with girls [4]. Autism spectrum disorder (ASD) is a persistent neurodevelopmental condition with early childhood onset. It begins in early childhood and persists throughout adulthood affecting three crucial areas of development: communication, social interaction, and creative or imaginative play [5]. The universal occurrence of autism spectrum disorders (ASD) was investigated about twenty-six years ago and it was thought to occur only in western industrialized countries that had high levels of technological development.

Over the years, knowledge about ASD and its prevalence has been documented as being on the rise in different regions of the world, with most literature coming from the western world, while the situation in Africa remains unclear [5]. No specific oral manifestations of ASD have been described, although the oral hygiene is known to be deficient [6]. Nevertheless, many authors have found the prevalence of caries and of periodontal disease to be no different compared with non-autistic individuals [6] and in some cases the prevalence of caries in children with ASD may even be comparatively lower [6]. Between $60-95 \%$ of all patients with ASD have an unusual sensory profile, including dysfunction in registering oral sensitivity. Studies have shown a high prevalence of periodontal and orthodontic problems such as crowding of the dentition in children with autism as compared to non-autistic children $[7,8]$. In addition, systemic medications taken by autistic patients may also affect their oral health. It has been reported that nearly $25 \%$ of young people with ASD present with gingival hyperplasia related to antiepileptic medications. Furthermore, some medication may compromise the health and function of the oral environment causing decreased salivary flow, dry mouth and a reduced mechanical and antibacterial action of saliva [8]. In recent years, there has been an increasing awareness of autism and autism-related diseases in the Cameroon. However, there is a paucity of information regarding the oral manifestations and the oral health care needs of children with ASD. The aim of the present study was to determine the prevalence of the oral manifestations in autism patients and to establish their oral health needs.

\section{Methodology}

This descriptive study was carried out in Yaoundé between January to July 2017 in all the specialized centers taking care of children with autism. These centers currently take care of children with ASD aged between 3-14 years regardless of gender. The eight centers identified were in five districts of the city of Yaoundé. Outside the Center for Special Education for children with ASD located in the district of Yaoundé I, the target population consisted of 145 children in four districts of the city of Yaoundé (II, IV, V, and VI). The districts of Yaoundé III and VII lacked education centers. Of the eight identified centers, seven agreed to participate in the study from the 4 districts based on the approval or consent of parents or guardians. Children aged between three and fourteen years old were included in the study. The inclusion criteria for participation in the study were defined before the sample were screened and consisted of the following: a diagnosis of ASD, the understanding of very simple instructions and written informed consent from the parents or caregivers. Data collection began following the approval by the relevant authorities as well as the consent from the parents and caregivers of the participants. Information from the patients was collected using a data capture sheet after which clinical examinations were carried out on a mobile dental clinic under bright natural light. The parents / caregivers of the participants received an explanation of the study, together with an information sheet and informed consent form. Those who agreed to participate completed a questionnaire evaluating the socio-demographic status of the children, medical history, functional disabilities, nutritional status, nutritional habits and oral hygiene of the patients. Data not adequately recorded were obtained by interviewing the parents or caregivers.

A single dentist was used to carry all the clinical dental examinations using a mirror and probe on a portable dental chair and guidable light source of enough power for adequate intraoral exploration. At the end of the examination, the parents and caregivers received a report on the oral disorders found, with suggestions for referral regarding the necessary treatments and dental care. Clinical examinations were carried out to evaluate the oral health status, the prevalence of dental caries, periodontal diseases, enamel hypoplasia, hard and soft tissues trauma and other pathologies in the oral cavity. The DMFT index was determined assessing the decayed, missing and filled teeth. Oral hygiene was rated using the Simplified Oral Hygiene Index (OHI-S). The criteria of this index are related to the sum of the Plaque Index Score (PI-S) and the Calculus Index Score (CI-S). In both cases the surfaces of index teeth were examined: vestibular surfaces of 11, 16, 26 and 31 ; lingual surfaces of 36 and 46. In the absence of index teeth, the entire sextant was recorded to the maximum degree (except for the third molars).

\section{Statistical Analysis}

Data were captured into Microsoft excel 2010 and exported to SPSS for statistical analysis. Results were presented in the form of tables using Microsoft Excel 2010. Bivariate analysis was carried out using Chi-square test for qualitative variables and ANOVA for quantitative variables $\mathrm{p}=0.05$.

\section{Ethical Considerations}

Approval to carry out this study was obtained from the Ethics Committee of the University des Montagnes. Informed consent was obtained from parents and all information obtained was strictly confidential. Participants were informed that they were free to leave the study at any stage without penalty to their further management.

\section{Results}

The sample consisted of ninety six children aged between 3 to 14 years. More than two thirds were male. Almost half 46 (47.9\%) were in the 3-7year age group while a more than a third 40 (41.7\%) were in the 11-14 year old age group. Children in Kindergarten were most represented (Table 1). 
Table 1: Socio-Demographic Characteristics.

\begin{tabular}{|cc|}
\hline Gender & Total n (\%) \\
\hline Male & $76(79.2)$ \\
Female & $20(20.8)$ \\
3-7 years & Age Range \\
7-11 years & $46(47.9)$ \\
11-14 years & $40(41.7)$ \\
& $10(10.4)$ \\
Kindergarten & Level of Education \\
Primary & $72(75.0)$ \\
Total & $24(25.0)$ \\
\hline
\end{tabular}

\section{Distribution of Bacterial Plaque and Gingivitis}

Large amounts of dental plaque deposits were found in half (45.21\%) of children 3 to 7 years and $44.41 \%$ between the ages of 7 to11 years had.

\section{Distribution of Dental Pathologies}

Gingival bleeding was found in $59.19 \%$ of the $7-11$ year old children, $55 \%$ in $3-7$ year old's and $16.14 \%$ in the $11-14$ year old children. Two thirds (66.13\%) of children aged between 7 and 11 years presented with dental caries Crown fracture (58.33\%) was found in more than half of the children aged between 3 and 7 years. Overall the mean DMFT index was 2.76, the plaque index was 3.91 and the gingival bleeding index 2.32. Enamel opacities were found in $51.43 \%$ in children aged 3 to 7 years, $45.71 \%$ aged between 7 and 11 years and $2.86 \%$ in the $11-14$ year old children). Enamel hypoplasia was identified in $42.86 \%$ in children aged 3 to 7 years, $50 \%$ in those between 7 and 11 years and $7.14 \%$ in the $11-14$ year old children. Macrodontia was found in $71.43 \%$ in children aged between 11 and 14 years) compared to $28.57 \%$ in those aged between 7 and 11 years. Orthodontic problems like malocclusion and crowding was found in $9.37 \%$ of the children. The prevalence of trauma to dental soft tissues was $45.83 \%$ while the prevalence of dental abnormalities such was 31\%. Nutritional practices, oral hygiene practices and oral health seeking behavior. More than two thirds $(62.5 \%)$ of the children were exposed to cariogenic foods. Tooth brushing for the majority (70.83\%) of the children was carried out by their mother. Half of the children brushed once a day. The majority brush their teeth before eating in the morning. Only 3 children brushed in morning and evening after meals. Two children reported brushing morning, afternoon and evening after meals. Only $13.54 \%$ had ever visited a dentist. Almost all $95.8 \%$ of the children had problems with word pronunciations. More than a third $38.5 \%$ of the children had difficulties talking aloud, a quarter had inconsistent speech (25\%) and a quarter with incoherence when singings $20.8 \%$. More than three quarters $(80.2 \%)$ of the children presented with dental sensitivity (Table 2). Almost all 93.8\% the children were not satisfied with their diet, $64.6 \%$ had difficulty with food intake (meals) and 62.5\% avoid eating (Table 3).
Table 2: Functional Disorders.

\begin{tabular}{|cc|}
\hline Speech Limitation & Frequency \\
\hline Inconsistent speech & $25.00 \%$ \\
Failure to answer questions & $15.60 \%$ \\
Incoherent singings & $20.80 \%$ \\
Shouting & $38.50 \%$ \\
Word Pronunciation Disorders & $95.80 \%$ \\
Oral Functional limitation & \\
Masticatory Difficulties & $24 \%$ \\
Associated Pathologies & \\
Dental pain & $16.70 \%$ \\
Dental Sensitivity & $80.20 \%$ \\
Bad Breath & $61.50 \%$ \\
\hline
\end{tabular}

Table 3: Dietary Behaviors.

\begin{tabular}{|cc|}
\hline Dietary Behavior & $\%$ \\
\hline Unsatisfactory diet & $93.80 \%$ \\
Difficulty with food intake (meals) & $64.60 \%$ \\
Avoid eating & $62.50 \%$ \\
No appetite & $13.50 \%$ \\
Reaction when eating & Agitated: $90.6 \%$ \\
& Calm: $9.4 \%$ \\
\hline
\end{tabular}

\section{Discussion}

Autism spectrum disorders (ASDs) comprise a complex set of related developmental disorders that are characterized by impairments in communication, social interaction, and repetitive behaviors. Impairments in sensory processing are also very common. The prevalence of ASD is increasing and is currently estimated to affect 1 in 150 children worldwide [9]. It has been documented in many areas of the world that ASD is dominant among male children $(3,4,5,6)$ as was found in the present study where there was a predominance of male children presenting with ASD. Various studies have reported the male: female ratio to range from 2:1 to 16:1 [9]. However, a recent systematic review carried out by Loomes R, in which fifty-four studies were analyzed, with $13,784,284$ participants of whom 53,712 had ASD $(43,972$ boys and 9,740 girls) concluded that the true male-to-female ratio is not $4: 1$ as is often assumed but rather, it is closer to 3:1. There appears to be a diagnostic gender bias meaning that girls who meet criteria for ASD are at disproportionate risk of not receiving a clinical diagnosis [10]. Whatever the true ratio, clinical referrals to a specialist diagnostic centre have seen a steady increase in the number of girls and women referred. Because of the male gender bias, girls are less likely to be identified with ASD, even when their symptoms are equally severe. Many girls are never referred for diagnosis and are missed from the statistics. Emphasis is placed on the different manifestations of behavior in autism spectrum conditions as seen in girls and women compared with boys and men [7].

The age group most represented in the present study population was between 3 and 7 years old. This could be explained by the fact that after the appearance of the first symptoms during 
the first three years of life, either from birth or shortly after 12 months [3]. The kindergarten age group was more dominant in our study as three quarters of the children attended kindergarten. This age group is very important in children with ASD as an important transition period. The kindergarten transition is the first major educational transition in a child's educational career. It is a particularly important transition for those with ASD because a successful kindergarten transition leads to better academic outcomes and better generalization of skills developed in the pre-school setting $[8,9]$. However, children with ASD begin with significant risk of an unsuccessful transition to kindergarten due to qualitative impairments in social communication and behavior. The use of recommended practices in kindergarten transition increases the chance of a successful transition for students with ASD $[8,9]$. Transitions in general, whether it be from one activity to another or one setting to another, are difficult for children with ASD [10]. These difficulties are often characterized by problems such as aggression, non-compliance, tantrums and a lack of consistent participation in activities [10]. It is because of these difficulties that the management of ASD is carried out using a multidisciplinary approach. The most effective strategies being based on educational programs $[6,10]$. In order to educate children with ASD, it is necessary to facilitate structured situations helping the patients to anticipate what is going to happen [6], since they show great fear and anxiety in the face of unknown situations [6], such as visiting the dental clinic. The dental care of these patients poses great difficulties, and in most cases, treatment is provided under general anaesthesia [6,11-16].

\section{Eating Habits and Oral Hygiene of Children ASD}

ASD are a major health and educational problem affecting many areas of daily living including eating. Children with ASD are often described as picky or selective eaters [12]. This was confirmed in the present study as two thirds of children preferred a soft and sweet diet. Poor reflexes during mastication and poor coordination of the tongue prevents normal swallowing and this results in a tendency to store food in the mouth and hence a preference for soft food. Various factors may contribute to food selectivity and several explanations have been proposed [12]. One of these factors relates to sensory sensitivity (also referred to as sensory defensiveness or sensory over-responsivity). Ayres [17] first described sensory defensiveness as the tactile domain (tactile defensiveness) in some children with learning and behavioral disorders. She described tactile defensiveness as an over-reaction to certain experiences of touch, often resulting in an observable aversion or negative behavioral response to certain tactile stimuli that most people would find innocuous. For example, children who show tactile defensiveness often have difficulty being cuddled and pull away from touch. It is possible that early tactile sensitivity may contribute to some of the sensory feeding issues such as difficulty with food textures seen in children with ASDs [12].

\section{Oral Hygiene Status and Practice}

The present study showed that the oral hygiene practices in terms of the frequency, duration and period of tooth brushing was very poor due to lack of co-operation, therefore the prevalence of dental caries and gingivitis is high. Parents and careers reported difficulties when they brushed the children's teeth [14] further compounded by the lack of manual dexterity of autistic children. Morales-Chávez (2017) found a lack of cooperation in daily dental hygiene in $35.7 \%$ of children with ASD. Maintaining good dental hygiene is a difficult task for 40 to $50 \%$ of people with ASD [15]. Rada (2010) similarly argues that lack of oral hygiene is one of the biggest causes of rapid increases in caries rates and periodontal disease in young people with ASD compared to neurotypical children [16]. In general, children preferred soft and sweetened foods, and tend to 'pouch' food inside the mouth instead of swallowing it due to poor tongue coordination, thereby increasing the susceptibility to caries $[18,19]$. Studies have shown that children with autism exhibited a higher caries prevalence, poor oral hygiene and extensive unmet needs for dental treatment than non-autistic children [19-21] this concurs with the present study where high levels of dental plaque was found. The children also presented with a high mean DMFT index, a high plaque index and a high gingival bleeding index and therefore require stringent oral hygiene practices. In the present study, oral hygiene practices were carried out mainly by the mothers of the children.

Vajawat and Deepika [19] suggested that attempts should be made by parents, general dentists and periodontists to teach oral hygiene methods to the children by constant repetition and patience, as autistic individuals can develop skills over a period and lead a more productive and independent life [19]. Jaber MA [18] also suggested that oral health programmed that emphasize prevention should be considered of importance for children and young people with autism [18]. Another reason for high prevalence of oral diseases in autism children is poor access to oral health care facilities. In the present study $86.46 \%$ of the children with ASD never consulted a dentist and this could be due to the lack of infrastructure and human resources to manage the children in Cameroon. Bartolomé Villar and colleagues [22] in their review, reported that certain disorders are more prevalent in association with ASD - malocclusions, enamel hypoplasia, parafunctional habits (bruxism) and deleterious habits (mouth breathing, tongue thrusting, rumination) as well as a higher incidence of dental trauma, owing either to accidents or to self-imposed injuries [22]. Similarly, in the present study, dental malocclusion, enamel hypoplasia and enamel opacities were observed among the children with ASD, as was bad breath. Motta et al. [23] found a statistically significant association between halitosis and mouth breathing [23]). In the present study the prevalence of dental trauma to both the hard and soft tissues was very high as compared to the case-control study carried out by Altun et al. [24] on ASD children (AG) and a control group (CG). They reported that the rate of injury was higher among the AG $(23 \%)$ than the CG $(15 \%)$, though the difference between the 2 groups, however, was not statistically significant $(\mathrm{P}<.19)$. The most common type of dental injury was enamel fracture. The rate of enamel fracture was higher in the CG (59\%) than in the AG (33\%), and the distribution of types of traumatic injury differed significantly between the AG and CG (P>.01). According to their study, the maxillary central incisors were most affected and enamel fracture was mostly common. This is because the maxillary central incisors are the more prominent and are the first teeth receive impact. In our study, the high level of injuries might because of the 
play grounds, or the surroundings of the children are not protected from activities that might prevent impactful contacts. In conclusion, children with autism presented with a high prevalence of dental caries and periodontal diseases, bad breath, and dental trauma to hard and soft tissues.

\section{Recommendation}

Since autism patients present with many functional and clinical problems, a multidisciplinary approach is needed in Cameroon for the management of both dental and behavioral problems.

\section{References}

1. Sheiham A (2005) Oral health, general health and quality of life. Bull World Health Org 83(9): 644.

2. (2017) Oral Health, General Health, and Well-Being. National Maternal and Child Oral Health Resource Center, Georgetown University, USA.

3. Newacheck PW, McManus M, Fox HB, Hung YY, Halfon N (2000) Access to health care for children with special health care needs. Pediatrics 105(4 Pt. 1): 760-766.

4. (2018) Estimated Prevalence of Children with Diagnosed Developmental Disabilities in the United States, 2014-2016. National Health Interview Survey of the USA.

5. Blomqvist M, Bejerot S, Dahllöf G (2015) A cross-sectional study on oral health and dental care in intellectually able adults with autism spectrum disorder. BMC Oral Health 15: 81.

6. Orellana LM, Silvestre FJ, Martínez Sanchis S, Martínez et al. (2012) Oral manifestations in a group of adults with autism spectrum disorder. Med Oral Pathol Oral Cir Bucal 17 (3): 415-419.

7. Bakare MO, Munir KM (2011) Autism spectrum disorders (ASD) in Africa: a perspective. Afr J Psychiatry 14(3): 208-210.

8. Luppanapornlarp S, Leelataweewud P, Putongkam P, Ketanont S (2010) Periodontal status and orthodontic treatment need of autistic children. World J Orthod 11(3): 256-261.

9. McCumber C (2011) Kindergarten transition practices for students with autism spectrum disorder. Thesis. Rochester Institute of Technology, USA.

10. McIntyre L, Eckert T, Fiese B, DiGennaro F, Wildenger L (2000) Transition to kindergarten: Family experiences and involvement. Early Childhood Education Journal 36: 83-90.
11. Peck A and Scarpati S (2000) Addressing the Needs of Students with Autism Spectrum Disorders. Teaching Exceptional Children 41: 4-13.

12. Cermak SA, Curtin C, Bandini LG (2010) Food selectivity and sensory sensitivity in children with autism spectrum disorders. J Am Diet Assoc 110(2): 238-246.

13. Loomes R, Hull L, Mandy WPL (2017) What Is the Male-to-Female Ratio in Autism Spectrum Disorder? A Systematic Review and Meta-Analysis. J Am Acad Child Adolesc Psychiatry 56(6): 466-474.

14. Ferrando Lucas MT, Martos Pérez J, Llorente Comí M, Freire Prudencio S, Ayuda Pascual R, et al. (2002) The autistic spectrum. An epidemiological study and analysis of possible subgroups. Rev Neurol 34 (Suppl 1): 4953.

15. Kamen S, Skier J (1985) Dental management of the autistic child. Spec Care Dentist 5: 20-23.

16. Dávila JM, Jensen OE (1988) Behavioral and pharmacological dental management of a patient with autism. Spec Care Dentist 8: 58-60.

17. Ayres AJ (1964) Tactile functions. Their relation to hyperactive and perceptual motor behavior. Am J Occup Ther 18: 6-11.

18. Jaber MA (2011) Dental caries experience, oral health status and treatment needs of dental patients with autism. J Appl Oral Sci 19(3): 212-217.

19. Vajawat M, Deepika PC (2012) Comparative evaluation of oral hygiene practices and oral health status in autistic and normal individuals. J Int Soc Prev Community Dent 2(2): 58-63.

20. Morales Chávez MC (2017) Oral Health Assessment of a Group of Children with Autism Disorder. J Clin Pediatr Dent 41(2): 147-149.

21. Rada RE (2010) Controversial issues in treating the dental patient with autism. J Am Dent Assoc 141(8): 947-953.

22. Bartolomé Villar B, Mourelle Martínez MR, Diéguez Pérez M, de Nova García MJ (2016) Incidence of oral health in paediatric patients with disabilities: Sensory disorders and autism spectrum disorder. Systematic review II. J Clin Exp Dent 8(3): 344-351.

23. Motta LJ, Bachiega JC, Guedes CC, Laranja LT, Bussadori SK (2011) Association between halitosis and mouth breathing in children. Clinics 66(6): 939-942.

24. Altun C, Guven G, Yorbik 0, Acikel C (2010) Dental injuries in autistic patients. Pediatr Dent 32(4): 343-346.

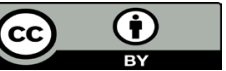

This work is licensed under Creative Commons Attribution 4.0 License

To Submit Your Article Click Here:

Submit Article
DOI: 10.32474/SJ0.2019.01.000123

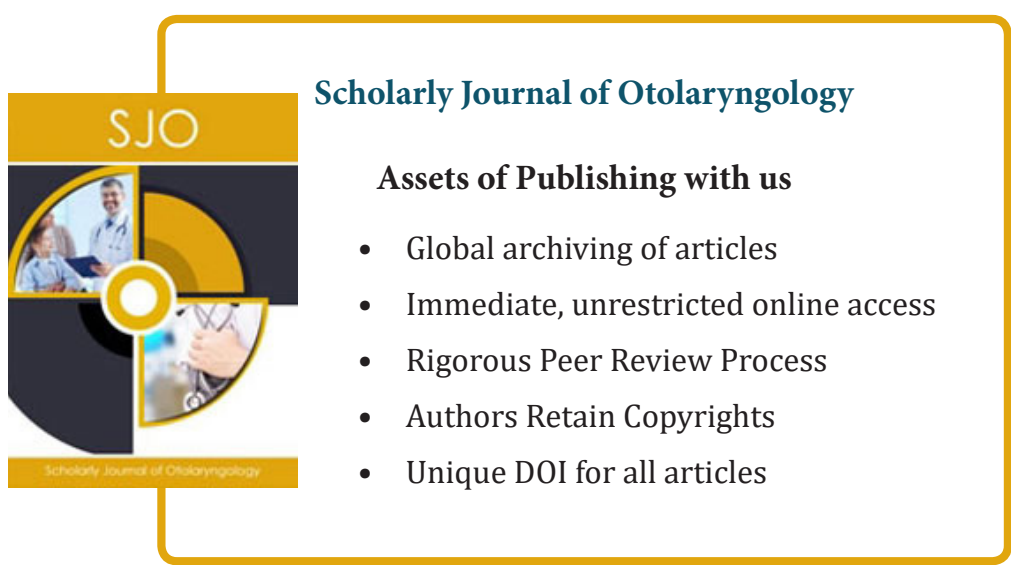

